\title{
Educación ambiental desde el trabajo comunal universitario: experiencia de la Reserva Biológica Alberto Manuel Brenes, San Ramón, Costa Rica
}

\author{
Environmental education from university communal work: \\ the experience of the Alberto Manuel Brenes Biological Reserve, \\ San Ramón, Costa Rica
}

Melvin Cartín Núñez

\author{
DOI: https://doi.org/10.22458/rb.v32i1.3550
}

Recibido- Received: 23/11/2020 / Corregido- Revised: 22/01/2021 / Aceptado- Accepted: 01/03/2021

\section{RESUMEN}

La acción social es un pilar fundamental del quehacer de la Universidad de Costa Rica. El trabajo comunal universitario forma parte de los programas de extensión universitaria y se caracteriza por alentar a las comunidades a ser autónomas en la búsqueda de soluciones para sus problemas cotidianos. El proyecto de educación ambiental de la Reserva Biológica Alberto Manuel Brenes tiene como objetivo contribuir al conocimiento del área protegida y de sus recursos naturales con el fin de promover su conservación. El proyecto inició sus labores en el 2010 y desde entonces más de 200 estudiantes se han matriculado, lo cual representa al menos 64000 horas de trabajo, en más de 50 centros educativos y similar número de comunidades. Gracias al proyecto, el número de personas que conocen la reserva biológica es indudablemente mayor que antes de su inicio.

Palabras clave: extensión universitaria; Costa Rica; recursos naturales; comunidades rurales.

\section{ABSTRACT}

Social action is an essential cornerstone in the work of the University of Costa Rica. The student community service is comprised within the university extension programs, and it is characterized by encouraging communities to become autonomous in the search of solutions to their everyday problems. The Environmental Education Project of the Alberto Manuel Brenes Biological Reserve aims at making the protected area and its natural resources known to people so as to promote its conservation. The project started in 2010 and since then more than 200 students have enrolled in it. This means that at least 64,000 hours of student community service have been carried out in more than 50 educational centers and a similar number of communities. Thanks to this project, the number of people who are now familiar with the biological reserve is undoubtedly higher than before its start.

Key words: university extension programs; Costa Rica; natural resources; rural communities.

* Profesor de Biología, Magíster en Desarrollo Sostenible. Universidad de Costa Rica, Sede de Occidente. Apartado 111-4250 San Ramón, Costa Rica. melvin.cartin@ucr.ac.cr

ID: https://orcid.org/0000-0002-2724-7498 


\section{Introducción}

La Universidad de Costa Rica (UCR) es una de las cinco universidades públicasde Costa Rica. La institución goza de reconocimiento dentro y fuera del territorio nacional (Vindas, 2016). Tres pilares fundamentales conforman su trabajo: docencia, investigación y acción social. De estos, es quizás la acción social el componente que menor atención recibe, mas no por ello menos importante.

La acción social (AS) se caracteriza por ser la actividad académica que vincula directamente a la Universidad con la sociedad. Es decir, a través de ella las diversas capacidades de la comunidad universitaria (docentes, estudiantes y personal administrativo) son puestas al servicio de las comunidades. Dentro de la AS se desarrollan gran cantidad de proyectos, organizados en tres grandes ejes: Extensión Cultural, Extensión Docente y Trabajo Comunal Universitario.

El Trabajo Comunal Universitario (TCU) se caracteriza por ser una actividad interdisciplinaria donde estudiantes y docentes de la UCR se acercan y trabajan en conjunto con las comunidades para atender diversos problemas sociales. No se trata de proyectos de asistencialismo, sino más bien de procesos que buscan la autonomía de las comunidades (UCR, 2017). Para ello, cada estudiante debe realizar $300 \mathrm{~h}$ de trabajo comunal como requisito para obtener su título de bachiller universitario, en cualquier disciplina académica.

Al tiempo que se trabaja en la resolución de problemáticas puntuales, el TCU les permite a los futuros profesionales sensibilizarse con los grupos más vulnerables de la población mediante el contacto directo y cotidiano. Lo anterior, contribuye a la formación humanista y crítica de los estudiantes quienes, en muchos casos, de no ser por el TCU desconocerían la realidad que enfrentan las comunidades, más allá del entorno propio de cada participante.

A inicios de 2021, la UCR contaba con 174 proyectos de TCU oficialmente inscritos (Ramírez, A., asesora de proyectos (Vicerrectoría de Acción Social, UCR), comunicación personal, 24 de febrero de 2021) a lo largo y ancho del país (incluso algunos empiezan a impactar más allá del territorio costarricense). Todos los proyectos nacen a partir de necesidades reales, cuya atención se considera prioritaria, ya sea por académicos o por miembros de la sociedad civil. Por tal razón, los proyectos no se circunscriben a unas pocas áreas temáticas, sino que abarcan prácticamente todo el quehacer de la realidad nacional, por ejemplo: educación, ambiente, exclusión social, pequeña empresa, patrimonio cultural, entre otros.

A pesar de lo anterior, muchos de los esfuerzos realizados a nivel del trabajo comunal pasan desapercibidos, incluso en la misma comunidad universitaria. Por tanto, el objetivo del presente trabajo es reseñar los principales antecedentes, resultados y logros alcanzados por el proyecto de educación ambiental en el Área de Amortiguamiento de la Reserva Biológica Alberto Manuel Brenes (ReBAMB), siglas institucionales TC-572, el cual está inscrito en la Sede de Occidente de la Universidad de Costa Rica.

\section{Antecedentes del proyecto}

El área protegida que hoy lleva el nombre de Reserva Biológica Alberto Manuel Brenes, cuya extensión es de 7800 ha (figura 1), nació como Reserva Forestal de San Ramón el 20 de junio de 1975, mediante el decreto 
ejecutivo 4960-A (Sistema Nacional de Áreas de Conservación -SINAC-, 2017). A inicios de la década de 1990, por medio del decreto 20172-M se cambió la categoría de reserva forestal por la de zona protectora, probablemente promovido por intereses de explotación minera en la zona (Salazar-Rodríguez, 2003). Su nombre y categoría actual surgieron gracias a la lucha de diferentes organizaciones, entre ellas la Sede de Occidente de la Universidad de Costa Rica, con lo cual en agosto de 1993 se publica la Ley 7354 que le confiere el estatus de reserva biológica.

\section{Figura 1}

Reserva Biológica Alberto Manuel Brenes y su área de amortiguamiento. Obsérvese la ubicación de algunas escuelas donde se desarrolla el proyecto de educación ambiental

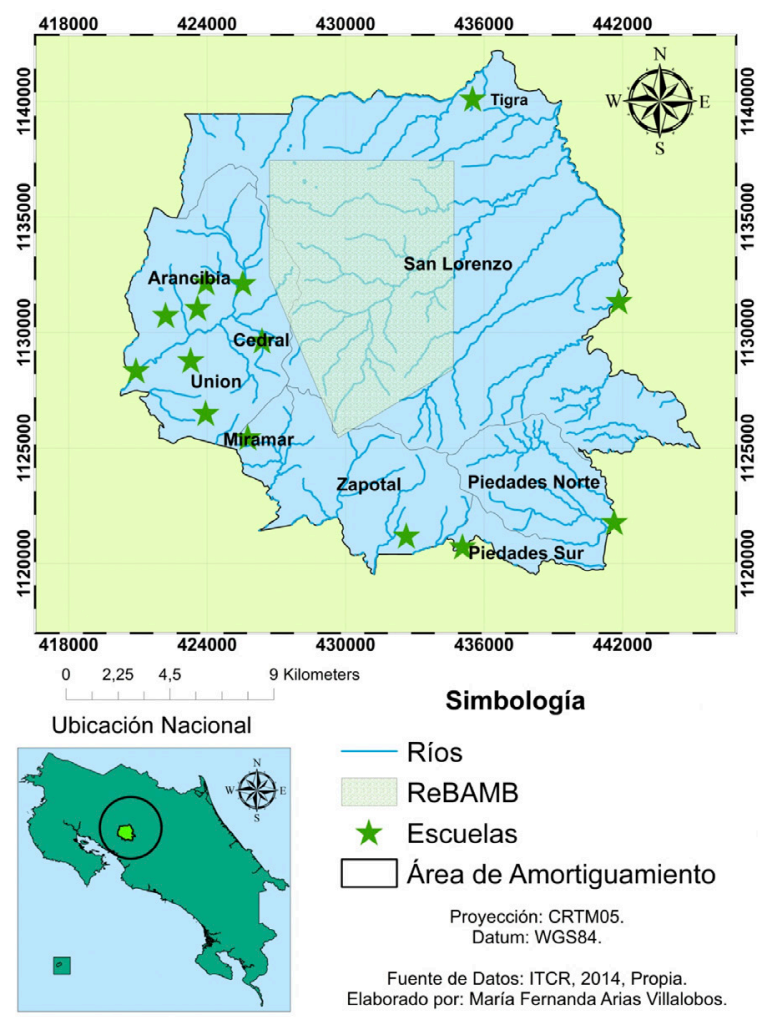

Nota. Mapa elaborado por Villalobos (2014), estudiante del TCU.
La ReBAMB presenta una particularidad: es la única área protegida que es coadministrada por una universidad pública, la Universidad de Costa Rica, Sede de Occidente (ubicada en San Ramón)y el Sistema Nacional de Áreas de Conservación (SINAC, 2017). Este modelo de administración compartida le confiere un carácter excepcional, incluso a nivel internacional (Salazar-Rodríguez, 2003), mismo que ha favorecido el desarrollo de la investigación, la docencia y la acción social en la ReBAMB y sus alrededores.

El proyecto de educación ambiental en el área de amortiguamiento de la ReBAMB surge en el año 2002, debido a la necesidad de proporcionar información básica sobre la importancia ecológica y económica de los recursos naturales protegidos por el AP (Barrantes, 2011). No obstante, durante algunos años no se logró concretar la consecución de jornada laboral para el proyecto, por tanto no se ejecutó.

En el año 2007, como resultado de una tesis de maestría (Guido, 2007),se conocieron una serie de indicadores socioambientales que evidenciaron la realidad particular de las comunidades aledañas a la Reserva Biológica Alberto Manuel Brenes (ReBAMB). Se encontraron buenos niveles en algunos indicadores, por ejemplo cobertura boscosa $(63,4 \%)$ y cobertura eléctrica ( $99 \%)$ pero al mismo tiempo otros indicadores resultaron muy preocupantes: altos niveles de desempleo $(24,6 \%)$ y altos niveles de pobreza $(73,3 \%)$. Otra de las conclusiones más preocupantes fue que el nivel de conocimiento de los pobladores con respecto a la ReBAMB era prácticamente nulo (Guido, 2007).

Sin duda, estos datos fueron determinantes para que en el 2010 iniciara la ejecución del proyecto de educación ambiental en las 
comunidades cercanas a la ReBAMB. En ese momento, el trabajo comenzó con una matrícula de casi 30 estudiantes, a cargo de la profesora Antonieta González y poco después de la profesora Teresa Barrantes, ambas docentes de la Sede de Occidente. A partir de ahí y de manera continua, el proyecto se ha mantenido activo en las comunidades del área de amortiguamiento e incluso más allá de las mismas.

\section{Metodología del proyecto}

Cuando los estudiantes universitarios alcanzan $50 \%$ de los créditos de su plan de estudios, están en posición de matricular su trabajo comunal. Para ello deben investigar la lista de proyectos abiertos y conocer cuáles de ellos abren espacios para su carrera de origen. Entre estos últimos, es el estudiante quien elige el proyecto que considera más conveniente.

En una primera etapa, los estudiantes matriculados en el proyecto TC-572 reciben una inducción del trabajo por realizar, del espacio geográfico de interés y de las principales metas propuestas. Luego se dividen en subgrupos de trabajo. El criterio para la conformación de los subgrupos es pertenecer a diferentes carreras para fomentar que el trabajo sea interdisciplinario.

En una segunda etapa, cada subgrupo somete a la consideración del encargado una propuesta de subproyecto, en la cual indican la comunidad o centro educativo donde van a trabajar, el objetivo, las metas y su respectivo cronograma de actividades. Una vez aprobado, la tercera etapa consiste en la ejecución de las actividades.

Lo anterior implica que en un mismo momento el proyecto TC-572 puede desarrollar actividades en diferentes instituciones y poblados. Como una forma de garantizar el éxito en las actividades propuestas, en cada institución o comunidad se ha contado con el apoyo de personas que actúan como enlace con la Universidad y que a su vez cumplen el papel de "contraparte" en la supervisión de los participantes. Así, en las instituciones educativas normalmente es el director quien asume el papel, mientras que en las comunidades han sido integrantes de asociaciones de desarrollo, de asociaciones administradoras de acueductos rurales (ASADAS) o incluso de organizaciones privadas sin fines de lucro, por ejemplo, grupos de adultos mayores.

Siendo niños y jóvenes el principal grupo meta del proyecto TC-572, se enfatiza en el uso de actividades lúdicas y participativas, donde el público no sea un mero espectador y receptor de información. Por eso las charlas, los talleres, los juegos y las dinámicas sonactividades comunes en las diferentes estrategias didácticas empleadas (cuadro 1). También se aprovecha el material audiovisual creado por los mismos participantes, cuyo contenido está relacionado con los diferentes recursos naturales protegidos en la reserva (Cartín, 2011; Jiménez, 2014; Araya et al., 2015).

Hasta el mes de enero de 2019, se habían matriculado 214 estudiantes pertenecientes a 20 carreras, de las cuales Gestión de Recursos Naturales ha aportado la mayor cantidad con 61 jóvenes y Preescolar con Concentración en Inglés la menor cantidad, con solamente una estudiante (figura 2). La mayoría de las carreras se imparten en la Sede de Occidente de la UCR, pero también se ha contado con participantes provenientes de otras sedes, en particular la Sede Rodrigo Facio (ubicada en San José, Costa Rica). En términos generales, lo citado representa más de 64000 horas de trabajo con las comunidades.

Es importante evidenciar que la mayoría de los estudiantes que matriculan el proyecto 


\section{Cuadro 1}

\section{Algunos ejemplos de actividades desarrolladas dentro de las estrategias didácticas}

de educación ambiental

\begin{tabular}{|c|c|c|c|}
\hline Temática & Tipo de actividad & Público meta & $\begin{array}{l}\text { Resultados esperados en función } \\
\text { de los participantes }\end{array}$ \\
\hline Generalidades de la ReBAMB & Charla & Adultos & Familiarizarse con la importancia del área protegida. \\
\hline Animales del bosque & $\begin{array}{c}\text { Cuento } \\
\text { musicalizado }\end{array}$ & Niños & $\begin{array}{c}\text { Conocer algunas de las especies más importantes de la } \\
\text { ReBAMB. } \\
\text { Sensibilizarse sobre el impacto de las actividades } \\
\text { humanas en la vida silvestre. }\end{array}$ \\
\hline Serpientes & Taller & Todo público & $\begin{array}{c}\text { Reconocer la importancia ecológica de las serpientes. } \\
\text { Identificar las especies más comunes y eventualmente } \\
\text { peligrosas. }\end{array}$ \\
\hline Áreas protegidas & Colocho (juego) & Niños & $\begin{array}{l}\text { Aprender el concepto de área protegida. } \\
\text { Asociar el uso permitido por el hombre con el área } \\
\text { protegida respectiva. }\end{array}$ \\
\hline Naturaleza & $\begin{array}{l}\text { Taller de haiku } \\
\text { (poesía) }\end{array}$ & Niños-jóvenes & $\begin{array}{l}\text { Expresar de forma creativa sus sentimientos hacia la } \\
\text { naturaleza (o sobre la ReBAMB). }\end{array}$ \\
\hline
\end{tabular}

Nota. Elaboración propia.

Figura 2

Carrera de procedencia de los estudiantes matriculados en el proyecto TC-572, período 2010-2019 $(n=214)$

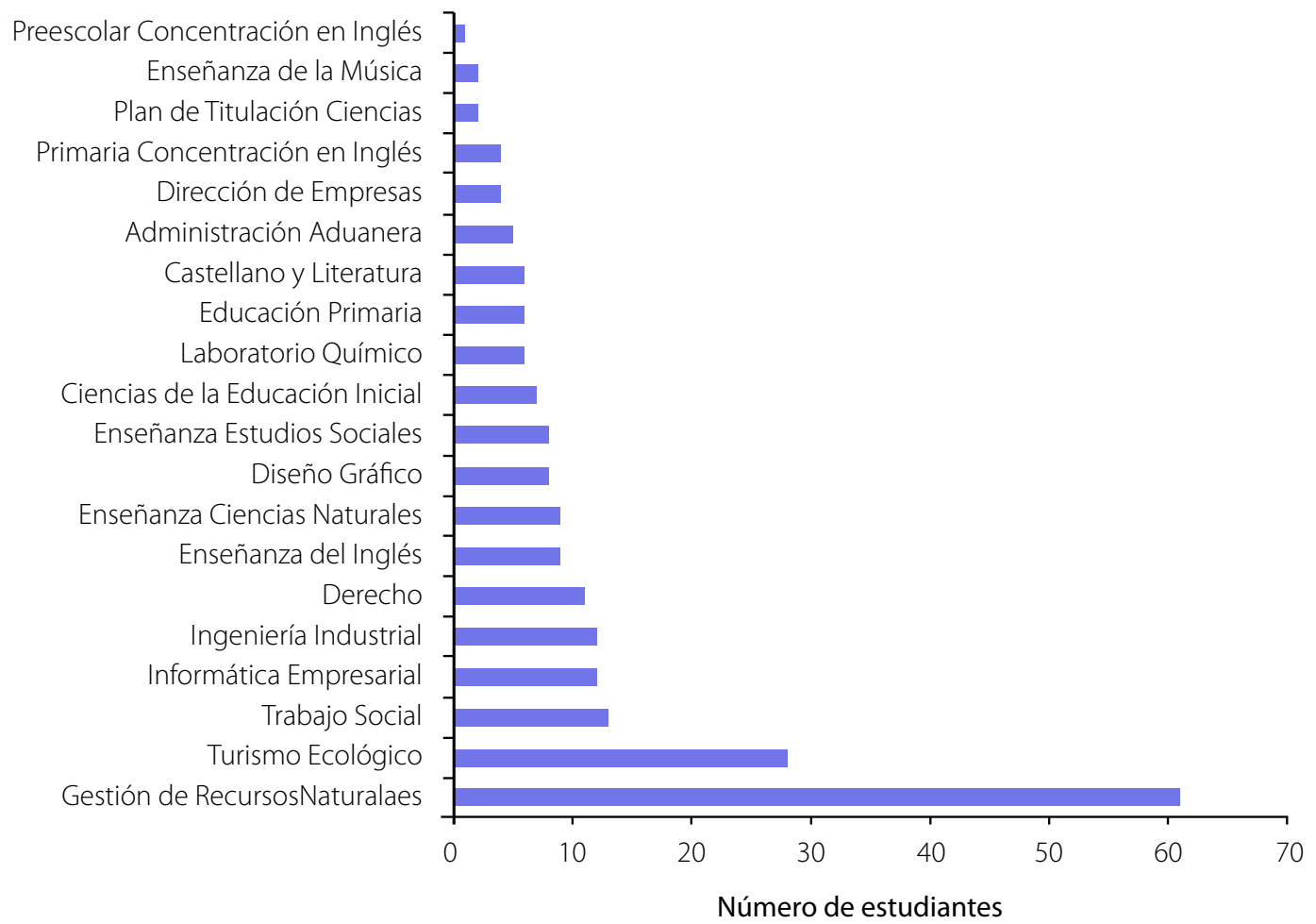

Nota. Elaboración propia. 
desconocen la ReBAMB. Por tal razón, antes de dar inicio al contacto con las comunidades, los propios jóvenes matriculados deben aprender sobre el área protegida y su importancia. Es decir, ellos son los primeros beneficiarios de la educación ambiental.Para ello, una de las principales actividades consiste en una gira de campo, en la cual conocen parte del área protegida y del trabajo cotidiano de funcionarios e investigadores de la ReBAMB.

\section{Centros educativos \\ y comunidades visitadas}

En el período 2010-2019 se han visitado más de 50 centros educativos (cuadro 2) ubicados en casi igual número de comunidades.
Los centros pertenecen a cuatro cantones, dos de la provincia de Alajuela (San Ramón y San Carlos) y dos de la provincia de Puntarenas (Puntarenas y Montes de Oro). Casi todas las instituciones son de tipo rural. Solamente el Instituto Julio Acosta (San Ramón) se encuentra en un área urbana así como la Escuela Alberto Manuel Brenes, que se ubica en la periferia de la ciudad de San Ramón, pero atiende una comunidad de alto riesgo social como lo es Bajo Tejares (Palma, 2013). Asimismo, de los 33 centros educativos visitados en la zona de trabajo, 14 son unidocentes, lo cual ejemplifica muy bien la "ruralidad" de las poblaciones donde se ha trabajado.

\section{Cuadro 2}

Centros educativos visitados, período 2010-2019. S= secundaria, $P M=$ primaria multidocente, $P U=$ primaria unidocente, $R=$ rural, $U=$ urbano, $K=$ preescolar

\begin{tabular}{|c|c|c|c|}
\hline Cantón/ Centro educativo & Comunidad & Tipo de centro & Tipo de población \\
\hline \multicolumn{4}{|c|}{ San Ramón } \\
\hline Centro Educativo El Socorro & Piedades Sur & PM & $\mathrm{R}$ \\
\hline CILEM & San Ramón & K & $R / U$ \\
\hline CINDEA & La Paz/Volio & S & $\mathrm{R}$ \\
\hline Colegio de Valle Azul & Valle Azul & S & $\mathrm{R}$ \\
\hline Colegio Técnico Profesional de Piedades Sur & Piedades Sur & PM & $\mathrm{R}$ \\
\hline Escuela Ángeles Norte & Ángeles Norte & PM & R \\
\hline Escuela Bajo Córdoba & Bajo Córdoba & PU & R \\
\hline Escuela Bajo La Paz & Bajo La Paz & PM & R \\
\hline Escuela de Coopezamora & Coopezamora & PM & R \\
\hline Escuela de Piedades Norte & Piedades Norte & PM & R \\
\hline Escuela de Potrerillos & Potrerillos, Piedades Sur & PU & $\mathrm{R}$ \\
\hline Escuela de San Isidro de Peñas Blancas & Peñas Blancas & PM & $\mathrm{R}$ \\
\hline Escuela de San Rafael & San Rafael & PM & $\mathrm{R}$ \\
\hline Escuela de San Rafael de Peñas Blancas & Peñas Blancas & PM & $\mathrm{R}$ \\
\hline Escuela de Santiago & Santiago & PM & $\mathrm{R}$ \\
\hline Escuela de Valle Azul & Valle Azul & PM & R \\
\hline
\end{tabular}


Escuela Ermelinda Mora

Escuela El Futuro

Escuela Federico Salas

Escuela Félix Ángel Salas

Escuela Fermín Rodríguez

Escuela Francisco J. Orlich

Escuela Gerardo Badilla

Escuela La Balsa

Escuela La Constancia

Escuela La Guaria

Escuela La Palma

Escuela Laboratorio

Escuela Los Criques

Escuela Los Lagos

Escuela Macario Valverde

Escuela Mercedes Quesada

Escuela Mons. Clodoveo Hidalgo

Escuela San Jorge

Instituto Julio Acosta García

Liceo de Magallanes

Liceo Nuestra Señora de Los Ángeles

Telesecundaria San Antonio de Zapotal
Cataratas, Alfaro

La Tigra

San Juan

Ángeles Sur

Bajo Rodríguez

Bajo La Paz

Alfaro

La Balsa

El Empalme

La Guaria, Piedades Sur

Piedades Sur

San Ramón

Los Criques

Los Lagos

Calle Zamora

Concepción

San Isidro

San Jorge

San Ramón

Santiago

San Ramón

Zapotal

San Carlos

Escuela La Tigra
PM

PM

PM

PM

PM

PM

PM

PU

PM

PM

PM

PM

PU

$P U$

PM

PM

PM

PU

$S$

S

S

S

\section{$\mathrm{R}$}

$\mathrm{R}$

$R / U$

R

$R$

$R$

$R / U$

R

$\mathrm{R}$

U

$\mathrm{R}$

U

R

$\mathrm{R}$

R

$\mathrm{R}$

$R$

$R$

S U

S R

$R / U$

$R$

\begin{tabular}{|c|c|c|c|}
\hline Escuela La Tigra & La Tigra & PM & $\mathrm{R}$ \\
\hline \multicolumn{4}{|c|}{ Montes de Oro } \\
\hline Escuela Corazón de Jesús & Corazón de Jesús & PU & $\mathrm{R}$ \\
\hline Escuela de Cedral & Cedral & PM & $\mathrm{R}$ \\
\hline Escuela Laguna & Laguna & PU & $\mathrm{R}$ \\
\hline Escuela Las Ventanas & Ventanas & PU & $\mathrm{R}$ \\
\hline Escuela Palmital & Palmital & PU & $\mathrm{R}$ \\
\hline Escuela San Buenaventura & San Buenaventura & PU & $R$ \\
\hline Escuela Tajo Alto & Tajo Alto & PU & $\mathrm{R}$ \\
\hline Escuela Zapotal & Zapotal & PU & $\mathrm{R}$ \\
\hline Liceo Rural de Cedral & Cedral & $S$ & $\mathrm{R}$ \\
\hline \multicolumn{4}{|c|}{ Puntarenas } \\
\hline Escuela Coopelagos & Coopelagos & PU & $\mathrm{R}$ \\
\hline Escuela de Arancibia & Arancibia & PU & R \\
\hline Escuela de Ojo de Agua & Ojo de Agua & PU & $R$ \\
\hline
\end{tabular}

Nota. Elaboración propia. 
Además de centros educativos, también se ha trabajado con otras instancias de la comunidad como asociaciones de desarrollo, ASADAS, comités de salud, grupos juveniles y grupos de adultos mayores (cuadro 3 ). Conservadoramente, se puede hablar de 5000 beneficiados directos, quienes a su vez han contribuido a propagar el conocimiento a familiares, vecinos y amigos. La temática de estas actividades siempre ha estado relacionada con la Reserva Alberto Manuel Brenes, desde generalidades de la misma, hasta aspectos más especíicos como los talleres sobre la importancia de ciertos grupos de animales, algunos considerados plagas y otros considerados peligrosos por las personas que conviven con ellos. Por ejemplo, entre los más solicitados se pueden mencionar talleres sobre serpientes y talleres sobre murciélagos (figura 3).

\section{Cuadro 3}

Algunos ejemplos de comunidades y actividades realizadas con otros sectores de la sociedad civil, período 2010-2019

\begin{tabular}{|c|c|c|c|}
\hline Lugar & Comunidad & Público meta & Temática \\
\hline ASADA de San Rafael & San Rafael, San Ramón & ASADA de San Rafael & Recurso hídrico \\
\hline ASADA de Santiago & Santiago, San Ramón & ASADA de Santiago & Recurso hídrico \\
\hline Biblioteca Pública de San Ramón & San Ramón & Adultos mayores & Informática y la ReBAMB \\
\hline Bosque Demostrativo UCR & Alfaro & Niños y jóvenes & Ecosistemas de la ReBAMB \\
\hline El Laguito UCR & San Ramón & Todo público & Información general \\
\hline Estadio Guillermo Vargas & San Ramón & $\begin{array}{l}\text { Escuela de fútbol Atlético } \\
\text { Ramonense }\end{array}$ & ReBAMB, vida silvestre \\
\hline Escuela de Potrerillos & Potrerillos & Finqueros & Convivencia pumas y finqueros \\
\hline Escuela de Zapotal & Zapotal & Asociación Las Orquídeas & Generalidades de la ReBAMB \\
\hline Escuela Fermín Rodríguez & Bajo Rodríguez & ASADAS & $\begin{array}{l}\text { Implicaciones de la nueva ley del recurso } \\
\text { hídrico }\end{array}$ \\
\hline Escuela Fermín Rodríguez & Bajo Rodríguez & Todo público & Feria ambiental \\
\hline Escuela Fermín Rodríguez & Bajo Rodríguez & $\begin{array}{l}\text { Personal de bares y } \\
\text { restaurantes }\end{array}$ & Manejo de desechos orgánicos \\
\hline Fundación Madre Verde & La Granja, Palmares & Todo público & ReBAMB, recurso hídrico \\
\hline Fundación SONATI & La Fortuna, San Carlos & Todo público & Felinos silvestres \\
\hline Hogar de Ancianos de San Ramón & San Ramón & Adultos mayores & $\begin{array}{l}\text { ReBAMB, recursos naturales, recurso } \\
\text { hídrico }\end{array}$ \\
\hline Museo de San Ramón & San Ramón & Niños y jóvenes & Vida silvestre y conservación de la ReBAMB \\
\hline Parque de Grecia & Grecia & Todo público & Información general \\
\hline Parque de Palmares & Palmares & Todo público & Información general \\
\hline Recinto de Tacares UCR & Tacares & Estudiantes universitarios & Información general \\
\hline Salón comunal de Bajo La Paz & La Paz & Asociación de desarrollo & Importancia de los murciélagos \\
\hline Salón comunal de Cedral & Cedral & Todo público & Feria ambiental \\
\hline Salón comunal de Cedral & Cedral & Todo público & Serpientes de la ReBAMB \\
\hline Salón comunal de Cedral & Cedral & Grupo de jóvenes & Generalidades de la ReBAMB \\
\hline
\end{tabular}

Nota. Elaboración propia. 
Figura 3

Taller de identificación de serpientes en la comunidad de Cedral de Miramar

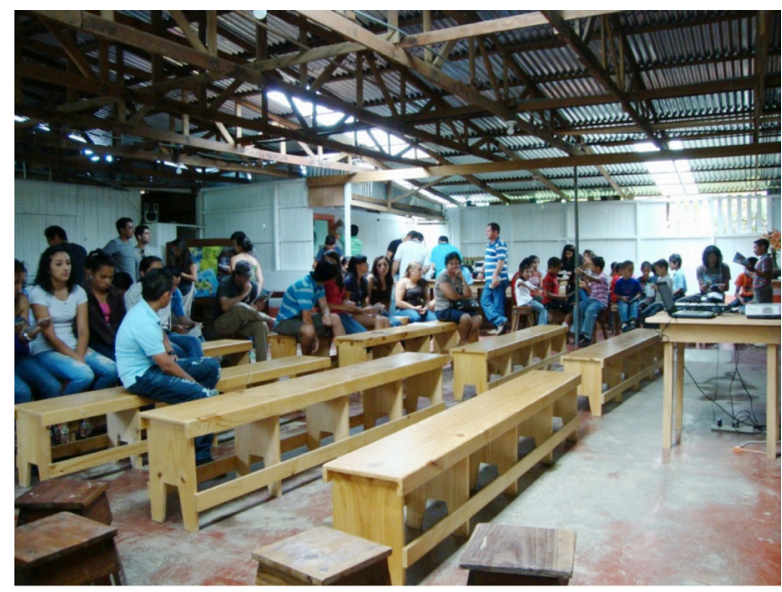

Nota. Fotografía de Teresa Barrantes, Montes de Oro, 2009.

\section{Conocimiento de la ReBAMB}

Como se mencionó anteriormente, a pesar de que la ReBAMB existe como área protegida desde la década de 1970, por años permaneció desconocida para muchos pobladores en su periferia. Por tanto, uno de los principales retos del TC-572 ha sido dar a conocer su existencia y su importancia. Así, luego de años de trabajo, se ha logrado pasar de un "conocimiento prácticamente nulo" (Guido, 2007) a alcanzar niveles de hasta $50 \%$ o más de los hogares (en algunos poblados) que ya reconocen la existencia del área protegida (figura 4). Lo anterior es un aspecto positivo, pero también indica que falta trabajo por hacer, dado que ninguna comunidad alcanza al menos $70 \%$, por tanto estarían "reprobando" en este apartado si hacemos la analogía con la nota mínima de aprobación en las materias universitarias.

Además, aunque afirman conocer la ReBAMB, el conocimiento sigue siendo confuso. Por ejemplo, ante la pregunta de si conocen alguna AP cercana a la comunidad, $65 \%$ de las personas entrevistadas respondieron que sí $(n=129)$. No obstante, al preguntar por el nombre del área protegida, cerca de 50\% de quienes respondieron afirmativamente $(n=83)$, contestaron no saber o simplemente no respondieron y solo $35 \%$ citó la ReBAMB (figura 5). Es decir, todavía la ReBAMB no es percibida como una vecina visible en algunas comunidades, lo cual es una gran debilidad.

\section{Figura 4}

Porcentaje de personas que manifestaron conocer la existencia de la Reserva Biológica Alberto Manuel Brenes, según la comunidad del entrevistado, enero $2014(n=127)$

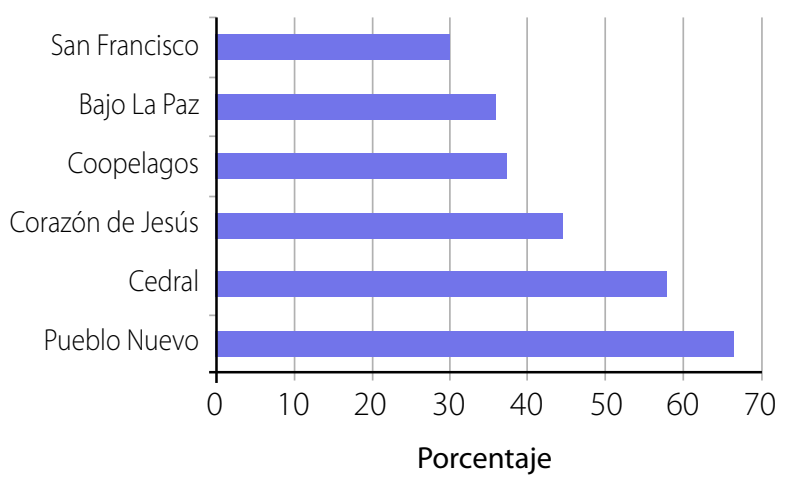

Nota. Elaboración propia con base en el estudio.

Figura 5

Respuestas dadas por los pobladores que mencionaron conocer la existencia de alguna área protegida cercana a su comunidad, enero $2014(n=83)$

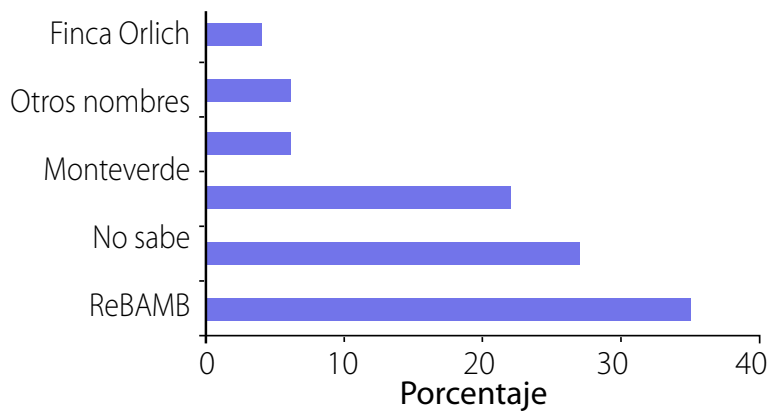

Nota. Elaboración propia con base en el estudio. 


\section{Plan de educación ambiental}

Por la dinámica interna de los proyectos de Acción Social de la UCR, el proyecto de Educación Ambiental TC-572 tiene que ser formulado y aprobado año tras año. No obstante, en el 2015, en conjunto con el Sistema Nacional de Áreas de Conservación, específicamente con la Administración de la Reserva Biológica Alberto Manuel Brenes, se decidió implementar un Plan de Educación Ambiental para un período de cinco años (2016-2020). El plan fue elaborado como parte de la práctica profesional de una estudiante de la carrera de Gestión de Recursos Naturales (Laura Quirós Elizondo), quien además era egresada del proyecto de TCU. El plan se propuso cubrir cinco ejes temáticos prioritarios: i) Mitigación y adaptación al cambio climático, ii) Manejo del recurso hídrico, iii) Producción sostenible, iv) La ReBAMB su importancia y prestación de servicios ecosistémicos y v) Relación ser humano-vida silvestre (Quirós, 2015).

Los ejes temáticos citados fueron considerados como prioritarios por diversos actores públicos y privados, entre ellos la Administración de la ReBAMB, el SINAC, la Municipalidad de Montes de Oro, miembros de COVIRENAS (Comités de Vigilancia de los Recursos Naturales), el Liceo Rural de Cedral, el Hotel Villa Blanca y el Bosque Eterno de los Niños. Para la ejecución del plan se decidió desarrollar un eje temático por año. Así, en el año 2016 se trabajó con La ReBAMB, su importancia y prestación de servicios ecosistémicos; en el 2017,con Manejo del recurso hídrico; en el 2018, la Relación ser humano-vida silvestre; en el 2019, el Cambio climático y para el 2020 se cerraría el primer plan con el tema Producción sostenible.

Ahora bien, el plan era dinámico. Esto quiere decir que si bien se propuso desarrollar un eje principal por año, las circunstancias podían provocar que se incluyeran otros temas. Por ejemplo, durante el 2016 e inicios del 2017, se reportaron varios incidentes de depredación de animales domésticos y de granja, causados por felinos silvestres en el área de interés. Por esta razón, el eje Relación ser humano-vida silvestre se empezó a desarrollar de manera simultánea con los otros ejes y en colaboración con otras instancias como es el caso de la Unidad de Atención de Conflictos con Felinos (UACFel-SINAC) y de la organización privada Panthera (figura 6).

\section{Figura 6}

Taller de medidas antidepredatorias en la comunidad de Potrerillos

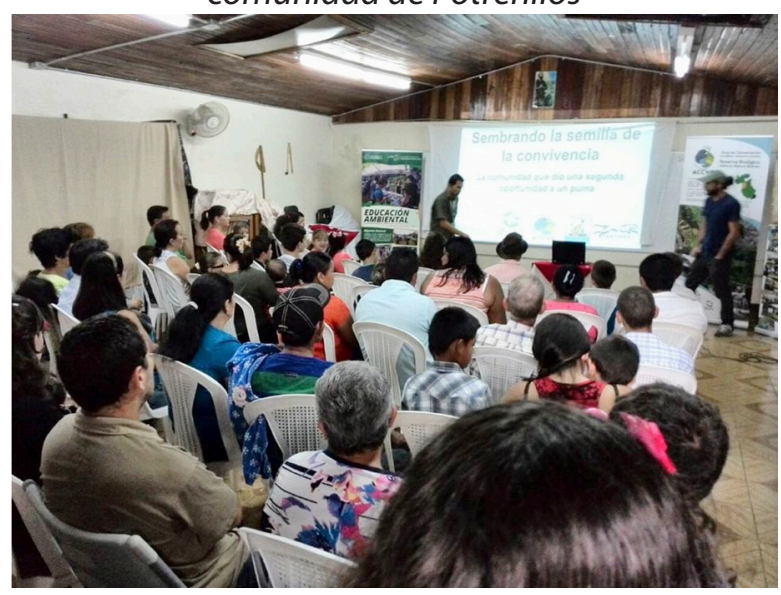

Nota. Fotografía de María José Arias. Piedades Sur de San Ramón, febrero de 2017.

El desarrollo de este plan ha sido un ejemplo exitoso del trabajo conjunto entre entidades públicas, como la Universidad de Costa Rica y el Sistema Nacional de Áreas de Conservación, así como entidades privadas (Panthera), que unen esfuerzos en procura de la conservación de los recursos naturales dentro y alrededor de un área protegida. Sin duda, uno de los principales retos es dar continuidad al esfuerzo conjunto, en especial para enfrentar amenazas como el cambio 
climático y también para demostrar a otras regiones que es posible la sana convivencia con la vida silvestre.

\section{Conclusiones}

El proyecto de trabajo comunal Educación ambiental en el área de amortiguamiento de la Reserva Biológica Alberto Manuel Brenes nació para subsanar el pobre conocimiento de los vecinos sobre el área protegida y la importancia de los recursos naturales presentes dentro y fuera de sus límites. Más de 200 estudiantes se han matriculado en el proyecto durante el período 2010-2019, lo cual representa un esfuerzo de aproximadamente $64 \mathrm{mil}$ horas de trabajo, principalmente en pueblos rurales de los cantones: San Ramón, San Carlos, Montes de Oro y Puntarenas. En estos años de trabajo el proyecto ha logrado llegar a más de 50 centros educativos y similar número de comunidades. Conservadoramente, se puede hablar de cinco mil beneficiados directos.

Aunque en casi todas las comunidades visitadas el número de personas que conocen sobre la ReBAMB y su importancia ha aumentado con el paso de los años, es necesario continuar el esfuerzo y trabajar con mayor intensidad en temáticas de interés socioambiental que se han detectado como prioridad, por ejemplo el cambio climático y la convivencia con la vida silvestre.

\section{Agradecimientos}

A todos los estudiantes $\mathrm{y}$ docentes universitarios que han trabajado en el TC-572. A la UCR, Sede de Occidente y a la Vicerrectoría de Acción Social. Al personal de la ReBAMB tanto de la UCR como del SINAC, y en especial a todas las comunidades que han abierto sus puertas desde el inicio del proyecto. 


\section{Referencias}

Araya, F., González, D., Soto, C. y Vargas, V. (2015). Información general de la ReBAMB. [DVD, Trabajo comunal universitario: Educación biológica y ambiental en el área de influencia de la Reserva Biológica Alberto Manuel Brenes. San Ramón, Costa Rica]. Universidad de Costa Rica.

Barrantes, T. (2011). Educación Biológica y Ambiental en el Área de Influencia de la Reserva Biológica Alberto Manuel Brenes en San Ramón, Alajuela. [Formulación de proyecto, Vicerrectoría de Acción Social]. Universidad de Costa Rica.

Cartín, M. (2011). Mamíferos de la Reserva Biológica Alberto MI. Brenes, San Ramón, Costa Rica. [DVD]. Universidad de Costa Rica.

Guido, I. (2007). Estimación del índice aproximado de sostenibilidad en la periferia de la Reserva Biológica Alberto Manuel Brenes, Costa Rica, 2007. [Tesis de maestría]. Universidad de Costa Rica.

Guido, I. (2009). Indicadores de sostenibilidad en los Procesos de conservación: El caso de la Reserva Biológica Alberto Manuel Brenes. Pensamiento Actual, 9 (12-13), 89-98.

Jiménez, J. (2014). Mamíferos de la Reserva Biológica Alberto Manuel Brenes. [Aplicación multimedia, Trabajo comunal universitario: Educación biológica y ambiental en el área de influencia de la Reserva Biológica Alberto Manuel Brenes]. Universidad de Costa Rica.
Palma-Villegas, C. (2013). Alfabetización de jóvenes y adultos mayores en la comunidad de Bajo Tejares, San Juan, San Ramón: una oportunidad para continuar aprendiendo. E-Ciencias de la Información, 3(1), 1-21. http://revistas.ucr.ac.cr/index.php/eciencias/article/view/8497/8020

Quirós, L. (2015). Plan de Educación Ambiental para la Reserva Biológica Alberto Manuel Brenes, Área de Conservación Cordillera Volcánica Central, Reserva de la Biosfera. [Práctica profesional]. Universidad de Costa Rica.

Salazar-Rodríguez, H. (2003). Reserva Biológica Alberto MI. Brenes: Una excepción en Costa Rica. Intersedes, V (8), 1-21. http://www.intersedes.ucr.ac.cr/ojs/index. php/intersedes/article/view/85/84

Sistema Nacional de Áreas de Conservación [SINAC]. (2017). Reserva Biológica Alberto Manuel Brenes. http://www.sinac.go.cr/ES/ac/accvc/rbamb/ Paginas/default.aspx

Universidad de Costa Rica (2017). Trabajo comunal universitario. https://accionsocial.ucr.ac.cr/tcu

Vindas, M. (2016, 16 de junio). UCR asciende en ranking de mejores universidades latinoamericanas. Portal de la investigación. https://vinv.ucr.ac.cr/noticias/ ucr-asciende-en-ranking-de-mejores-universida$\underline{\text { des-latinoamericanas }}$ 